\title{
GNSS and SAR Signal Delay in Perturbed Ionospheric D-Region During Solar X-Ray Flares
}

\author{
Aleksandra Nina ${ }^{(}$, Giovanni Nico $^{(}$, Senior Member, IEEE, Oleg Odalović, Vladimir M. Čadež, \\ Miljana Todorović Drakul, Milan Radovanović, and Luka Č. Popović
}

\begin{abstract}
We investigate the influence of the perturbed (by a solar X-ray flare) ionospheric D-region on the global navigation satellite systems (GNSS) and synthetic aperture radar (SAR) signals. We calculate a signal delay in the D-region based on the low ionospheric monitoring by very-low-frequency (VLF) radio waves. The results show that the ionospheric delay in the perturbed D-region can be important and, therefore, should be taken into account in modeling the ionospheric influence on the GNSS and SAR signal propagation and in calculations relevant for space geodesy. This conclusion is significant because numerous existing models ignore the impact of this ionospheric part on the GNSS and SAR signals due to its small electron density which is true only in quiet conditions and can result in significant errors in space geodesy during intensive ionospheric disturbances.
\end{abstract}

Index Terms-Global navigation satellite systems (GNSS), ionosphere, synthetic aperture radar (SAR) interferometry (InSAR), very-low-frequency (VLF) radio signals.

\section{INTRODUCTION}

$\mathbf{N}$ OWADAYS spaceborne measurements of positioning, displacements, navigation, and timing play an important and critical role in telecommunications, geodesy, all forms of transportation and other human activities. These measurements are primarily provided by the global navigation

Manuscript received September 20, 2018; revised February 6, 2019 and July 15, 2019; accepted September 4, 2019. This work was supported by the Ministry of Education, Science and Technological Development of the Republic of Serbia under Projects 176001, 176002, 176004, III44002, III47007, and TR36020, and the OT4CLIMA Project which was funded by the Italian Ministry of Education, University and Research (D.D. 2261 del 6.9.2018, PON R\&I 2014-2020, and Fondo per lo Sviluppo e la Coesione). (Corresponding author: Aleksandra Nina.)

A. Nina is with the Institute of Physics Belgrade, University of Belgrade, 11080 Belgrade, Serbia (e-mail: sandrast@ipb.ac.rs).

G. Nico is with the Istituto per le Applicazioni del Calcolo (IAC), Consiglio Nazionale delle Ricerche (CNR), 70126 Bari, Italy, and also with the Department of Cartography and Geoinformatics, Institute of Earth Sciences, Saint Petersburg State University (SPSU), 199034 Saint Petersburg, Russia (e-mail: g.nico@ba.iac.cnr.it).

O. Odalović and M. Todorović Drakul are with the Department of Geodesy and Geoinformatics, Faculty of Civil Engineering, University of Belgrade, 11000 Belgrade, Serbia.

V. M. Čadež is with the Astronomical Observatory, 11060 Belgrade, Serbia. M. Radovanović is with the Geographical Institute "Jovan Cvijić" SASA, 11000 Belgrade, Serbia, and also with the Institute of Sports, Tourism and Service, South Ural State University, 454080 Chelyabinsk, Russia.

L. C. Popović is with the Astronomical Observatory, 11060 Belgrade, Serbia, and also with the Faculty of Science, University of Banja Luka, 78000 Banja Luka, Bosnia and Herzegovina.

Color versions of one or more of the figures in this letter are available online at http://ieeexplore.iee.org.

Digital Object Identifier 10.1109/LGRS.2019.2941643 satellite systems (GNSS) and synthetic aperture radar (SAR) interferometry (InSAR). In the case of GNSS signals, the accuracy of positioning depends on both the errors in measurements and the way of modeling different influences on signal propagation. There are many sources causing these errors and their modeling indicates various effects on cumulative error. The methodology presented in this article is intended to be applied to single-frequency GNSS receivers. For these receivers, one should know the precise satellite orbits, satellite clock errors and hardware delay, receiver hardware, ionospheric and tropospheric delay, multipath and phase center variation which delays are $2.5-5 \mathrm{~cm}$, up to $2 \mathrm{~cm}$, up to $3 \mathrm{~m}$, 2.3-5 m, 6 and up to $1 \mathrm{~cm}$, respectively [1]. This indicates that sources contributing errors of already $1 \mathrm{~cm}$ have to be included in calculations of the GNSS positioning. As far as InSAR is concerned, the measurement of displacements (landslides, earthquakes, glaciers, and infrastructures) with a subcentimetric precision requires the knowledge of precise orbits, high-resolution digital elevation models and good modeling of propagation effects in the atmosphere, including both troposphere and ionosphere [2], [3]. Recently, the potential of InSAR to provide maps of tropospheric and ionospheric delays with high spatial resolution, even for low temporal sampling, was proven [4], [5]. In particular, SAR meteorology is complementing the techniques of GNSS meteorology [6] and GNSS tomography (see [7]) to measure the atmospheric water vapor.

The ionosphere can be a significant source of delay for the GNSS and SAR signals. This deviation is caused by free electrons whose density is significantly larger in the upper ionosphere. For this reason, modeling of the ionospheric influences on satellite signals is often based on the analyses for altitudes above $90 \mathrm{~km}$. Also, even a model includes the D-region $(60-90 \mathrm{~km})$ in calculations, input parameters obtained in observations are relevant for one or a few altitudes above this layer, and a part of the total electron content (TEC) in the D-region is calculated using extrapolation with the unique expressions for all conditions including cases when perturbations are dominant or only exist below $90 \mathrm{~km}$. However, this limitation/extrapolation (applicable to the quiet conditions) opens a question: Can the perturbed D-region sufficiently affect the GNSS and SAR signals so that the inclusion of the perturbed D-region observation data in models becomes necessary for measurements? 
Lack of studies directed to research of these approximations motivated us to study the influence of the perturbed D-region on GNSS and SAR signals. We consider perturbations induced by a solar X-ray flare which is one of the phenomena whose occurrence primarily disturbs the low ionosphere and can cause an increase of the electron density by one or two orders of magnitude in the D-region [8], [9].

There are two goals of this study. First, we investigate the properties of the time evolution of the ionospheric delay in the $\mathrm{D}$-region during a solar X-ray flare. The fact that X-ray flares induce similar properties of the D-region electron density time and space distributions (see [10]) which are required for delay calculations allows us to use one solar X-ray flare as an example (appeared on May 1, 2013). Second, we analyze the D-region delay dependencies from the $\mathrm{X}$ radiation flux maximum using observations given in [11].

\section{IONOSPHERIC DELAY: DifFERENT CONTRIBUtions}

The ionospheric influence on the GNSS/SAR signal deviation can be described by the ionospheric delay $P_{\mathrm{I}}=\alpha_{\mathrm{I}} \mathrm{TEC} / f^{2}$ where $\alpha_{\mathrm{I}}=40.3 \mathrm{~m}^{3} \mathrm{~s}^{-2}$, and TEC $=\int_{l_{\mathrm{I}}} N_{\mathrm{e}} d l_{\mathrm{I}}$ is the TEC (in $\mathrm{m}^{-2}$ ) along the ray path of length $l_{\mathrm{I}}$ in the ionosphere with the electron density $N_{\mathrm{e}}$.

Determination of TEC is not a simple task because of a nonstationary and complex electron density spatial distribution. The F-region has the most important influence on $P_{\mathrm{I}}$ and the contribution of other layers decrease with altitude. For this reason, TEC is generally calculated by single-layer models (SLM) which assume the entire effect localized to some fixed height in the F-region [12], [13], or multiple-layer models (MLM) which used observation data at the ionospheric E- and F-region height even the D-region contribution is included in total TEC [14]- [16]. However, the ionosphere is under permanent influences of ionizing radiation from the outer space and terrestrial layers which can significantly increase the electron density and result in the increase of the ionospheric influence on the GNSS/SAR signals [17], [18]. The importance of these effects varies with altitude which can indicate a need for additional measurements modeling.

\section{Modeling of the D-Region Delay}

Like for the entire ionosphere, the D-region delay can be expressed as

$$
P_{\mathrm{D}}=\alpha_{\mathrm{I}} \mathrm{TEC}_{\mathrm{D}} / f^{2} .
$$

The $\mathrm{TEC}_{\mathrm{D}}=\int_{l_{\mathrm{D}}} N_{\mathrm{e}} d l_{\mathrm{D}}$ for the signal propagation path $l_{\mathrm{D}}$ in the D-region can be obtained from models of the electron density space and time distributions $N_{\mathrm{e}}$, and the considered signal propagation path.

\section{A. D-Region Electron Density}

Determination of the electron density is a very complex issue for both in situ measurements by rockets and remote sensing by radio waves. The reasons are the absence of sudden event detections as the measurements are not continuous in time, medium perturbations caused by the moving rocket which creates errors in obtained quantities and lack of knowledge of numerous parameters which are usually unavailable for the considered location during the observation. Nowadays, the radio signals are most frequently utilized in the low ionospheric monitoring which provides necessary data for various models to compute the D-region plasma parameters during perturbations induced by different sudden events.

In this letter, determination of the electron density during an $\mathrm{X}$-ray flare is achieved by a procedure [19] based on matching changes of the observed very-low-frequency (VLF) signal (its amplitude $\Delta A$ and phase $\Delta P$ ) with corresponding values resulting from simulations of the VLF signal propagation using the long-wave propagation capability (LWPC) numerical model developed by the Naval Ocean Systems Center, San Diego, USA [20]. The simulated values of amplitude and phase are obtained by varying two independent parameters, the "sharpness" $\beta$ and the signal reflection height $H^{\prime}$, which characterize Wait's model of the ionosphere [21]. Finally, the values of parameters that yield the best fit of simulated values with experimental data are used to determine the electron density according to the equation for the horizontal uniform ionosphere [22]

$$
N_{\mathrm{e}}(h, t)=1.43 \cdot 10^{13} e^{-\beta(t) H^{\prime}(t)} e^{(\beta(t)-0.15) h}
$$

which is commonly used for the D-region electron density modeling in numerous papers [9], [10], [23]. Here, $N_{\mathrm{e}}, \beta$ and $H^{\prime}$ are given in $\mathrm{m}^{-3}, \mathrm{~km}^{-1}$, and $\mathrm{km}$, respectively.

It is worth noting that the form of (2) depends on the ionosphere model. Differences among models are more pronounced both at higher D-region altitudes and for more intense solar X-ray flares [24]. For these reasons, we consider flares of classes lower than M5, and compare results for the entire D-region $(60-90 \mathrm{~km})$ with those for a reduced altitude range below $80 \mathrm{~km}$, where the expected influence of model selection is less pronounced.

\section{B. GNSS/SAR Signal Propagation Path in the D-Region}

The D-region of thickness $H_{\mathrm{D}}$ is modeled as a set of $N_{\mathrm{D}}$ horizontally uniform layers of thickness $\delta=H_{\mathrm{D}} / N_{\mathrm{D}}$. The GNSS/SAR signal refracts at the $i$ th layer according to the refractive index $n_{\mathrm{i}}$ [25]

$$
n_{\mathrm{i}}=\sqrt{1-f_{\mathrm{pi}}^{2} / f^{2}}
$$

where $f$ is the signal frequency, $f_{\mathrm{pi}}=8.98\left(N_{\mathrm{ei}}\right)^{1 / 2}$ (in $\mathrm{Hz}$ ) is the electron plasma frequency at the $i$ th horizontal uniform layer with electron density $N_{\mathrm{ei}}$ (in $\mathrm{m}^{-3}$ ) centered around the height $h_{i}$ related to the middle of the $i$-th layer. Here, we notice that the collision frequency is ignored because calculations showed its negligible influence on the refractive index. Refraction on the boundaries can be described by Snell's law [25]

$$
n_{\mathrm{i}} \sin \left(\Theta_{\mathrm{i}}\right)=n_{\mathrm{i}-1} \sin \left(\Theta_{\mathrm{i}-1}\right)=n_{0} \sin \left(\Theta_{0}\right)
$$

where $i=1,2, \ldots, \mathrm{N}_{\mathrm{D}}+1$ and $\Theta_{i}$ is the wave propagation angle in the $i$ th layer. According to (4) in individual layer $i$ of thickness $\delta H_{\mathrm{D}}$, the wave path length $l_{\mathrm{i}}=\delta H_{\mathrm{D}} / \cos \left(\Theta_{\mathrm{i}}\right)$ is

$$
l_{i}=n_{\mathrm{i}} \delta H_{\mathrm{D}}\left[n_{\mathrm{i}}{ }^{2}-\left(n_{0} \sin \left(\Theta_{0}\right)\right)^{2}\right]^{-0.5}
$$




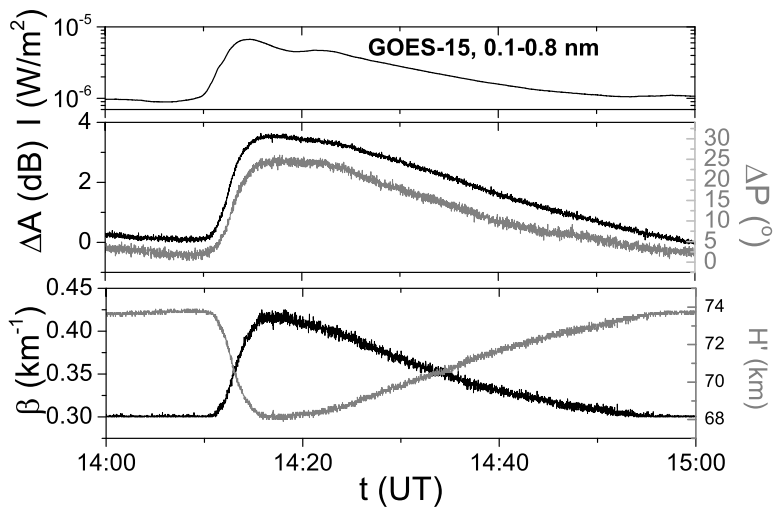

Fig. 1. (Top) Time dependencies of the X-ray radiation flux recorded by the GOES-15 satellite. (Middle) Signal amplitude and phase recorded in Serbia. (Bottom) Wait's parameters calculated by the procedure given in Section III.

where $\Theta_{0}$ and $n_{0}$ are the incident angle of the GNSS/SAR signal in the D-region and refractive index of the upper layer.

\section{C. $P_{D}$ Modeling}

In this study, we derived expression for calculation of the D-region delay using the above explained model for the electron density calculation (Section III-A) and presented procedure for the GNSS/SAR signal path determination (Section III-B). From (5) and $\mathrm{TEC}_{\mathrm{D}}=\sum_{i=1}^{N_{\mathrm{D}}} N_{\mathrm{ei}} \mathrm{l}_{\mathrm{i}}$, we first obtain

$$
\mathrm{TEC}_{\mathrm{D}}=\delta H_{\mathrm{D}} \sum_{i=1}^{N_{\mathrm{D}}} N_{\mathrm{ei}} n_{\mathrm{i}}\left[n_{\mathrm{i}}{ }^{2}-\left(n_{0} \sin \left(\Theta_{0}\right)\right)^{2}\right]^{-0.5} .
$$

Finally, knowing the incident angle of the GNSS/SAR signal in the D-region, $\Theta_{0}$, and altitude distributions of the electron density $N_{\mathrm{e}}$ necessary for calculation of the refractive index according (3), we express $P_{\mathrm{D}}$ from (1) and (6)

$$
P_{\mathrm{D}}=\frac{C \delta H_{D}}{f^{2}} \sum_{i=1}^{N_{\mathrm{D}}} N_{\mathrm{ei}} n_{\mathrm{i}}\left[n_{\mathrm{i}}^{2}-\left(n_{0} \sin \left(\Theta_{0}\right)\right)^{2}\right]^{-0.5}
$$

for the modeled D-region by $N_{\mathrm{D}}$ uniform layers with depth $\delta H_{\mathrm{D}}$. We notice that, in the cases of local perturbations, the used approximation of horizontal uniform layers is not possible and more data sources need to be included in modeling.

\section{OBSERVATIONS AND DATA}

The first part of our analysis is directed to ionospheric perturbations induced by the solar X-ray flare of May 1, 2013. The increase of photon flux recorded by the National Oceanic and Atmospheric Administration (NOAA) satellite GOES-15, and its time evolution within the wavelengths range $0.1-0.8 \mathrm{~nm}$ is shown in Fig. 1 (top).

Data for the low ionospheric modeling were obtained using the 23.4-kHz VLF signal emitted by the DHO transmitter located in Rhauderfehn (Germany) and received at the Institute of Physics in Belgrade (Serbia). This transmitter was chosen because it provides a relatively short signal propagation path as well as the most suitable signal characteristics for the location of the receiver [26]. The ionospheric perturbations

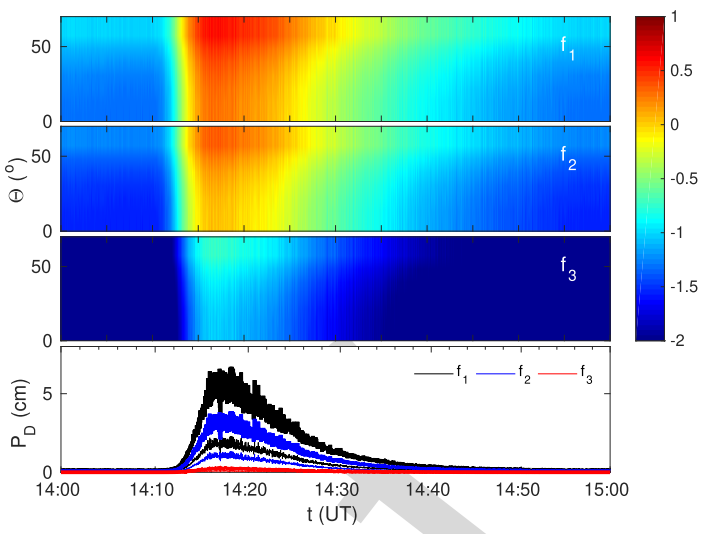

Fig. 2. (Top three panels) Time evolutions of $\log \left(P_{\mathrm{D}} / 1 \mathrm{~cm}\right)$ for incident angles $\Theta_{0}$ in the range $0^{\circ}-70^{\circ}$ and GNSS/SAR frequencies $f_{1}=1.20000$ $\mathrm{GHz}, f_{2}=1.57542 \mathrm{GHz}$, and $f_{3}=5.40500 \mathrm{GHz}$. (Bottom panel) Time evolutions of $P_{\mathrm{D}}$ for incident angles of $0^{\circ}$ and $70^{\circ}$ for the considered frequencies.

were detected as the amplitude $\Delta A$ and phase $\Delta P$ variations of the considered VLF signal Fig. 1 (middle) recorded by the Absolute Phase and Amplitude Logger (AbsPAL) VLF receiver. The Wait's parameters $\beta$ and $H^{\prime}$, as shown in Fig. 1 (bottom), are obtained by applying the numerical procedure described in Section III to these data. A good agreement was found with results presented in [10], [11], and [19]. Their implementation in analytical expressions also given in Section III provides the time evolution of the D-region time delay.

In the second part of this study, we used data from literature which are also obtained from the low ionospheric observations by the VLF signals [11].

\section{RESULTS AND DisCUSSION}

The procedure given in Section III is applied to the data recorded by the Belgrade very low/low frequency (VLF/LF) receiver station: at the time of solar X-ray flare of May 1, 2013 (the first part of this study) and at the time of maximum flux of solar X-ray flares considered in [11]. In both parts, we considered three GNSS/SAR frequencies $f_{1}=1.2 \mathrm{GHz}$ (GNSS, ALOS-2 or the future NISAR mission), $f_{2}=1.57542 \mathrm{GHz}$ (GNSS), and $f_{3}=5.4 \mathrm{GHz}$ (Sentinel-1 and Radarsat-2) with incident angles $\Theta_{0}$ between $0^{\circ}$ and $70^{\circ}$ (relevant for the satellite positioning).

\section{A. Time Evolution of $P_{\mathrm{D}}$-Example of Particular Event}

A visualization of influences of the GNSS/SAR signal incident angle $\Theta_{0}$ and the signal frequency $f$ to the D-region delay $P_{\mathrm{D}}$ during the considered event is shown in Fig. 2. As one can see the D-region influence increases with incident angle due to increasing of signal propagation path within the D-region and, consequently, $\mathrm{TEC}_{\mathrm{D}}$ (see Section III). It is lowest for the signal with frequency $f_{3}$ which is the highest considered frequency. The decrease of $P_{\mathrm{D}}$ with frequency can be explained by (7), which indicates that $P_{\mathrm{D}} \sim f^{-2}$. The other two frequencies show very similar time evolutions of $P_{\mathrm{D}}$.

The time evolutions of $P_{\mathrm{D}}$, as shown in Fig. 2, illustrate that the changes of $P_{\mathrm{D}}$ reach more than two orders of magnitude 


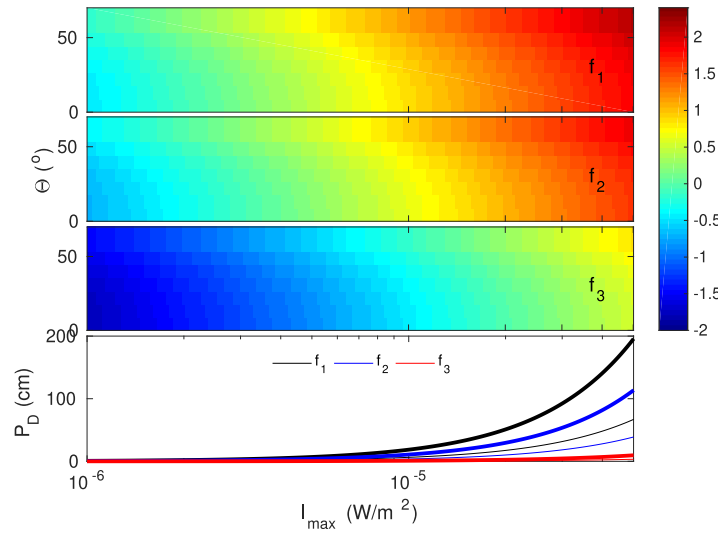

Fig. 3. (Top three panels) $P_{\mathrm{D}}$ dependence on $I_{\max }$ for incident angles $\Theta_{0}$ in the range $0^{\circ}-70^{\circ}$ and GNSS/SAR frequencies $f_{1}=1.20000 \mathrm{GHz}$, $f_{2}=1.57542 \mathrm{GHz}$, and $f_{3}=5.40500 \mathrm{GHz}$. (Bottom panel) Time evolutions of $P_{\mathrm{D}}$ for incident angles of $0^{\circ}$ and $70^{\circ}$ for the considered frequencies.

larger values when the considered flare event is present. These values reach a few centimeters which, keeping in mind that errors of about $1 \mathrm{~cm}$ are already considered nonnegligible in modeling, clearly indicates the significance of the role the perturbed D-region plays in accuracy of the GNSS/SAR measurements during a solar X-ray flare activity. In this figure, it is visible that time period with $P_{\mathrm{D}}>1 \mathrm{~cm}$ depends on the considered frequency $f$ (it decrease with frequency because decreasing of $P_{\mathrm{D}}$ ) and angle $\Theta_{0}$ (it increase with $\Theta_{0}$ due to increasing of $P_{\mathrm{D}}$ in time), and that this period can take several tens of minutes. Keeping in mind that the considered X-ray solar flare is not so strong (the C-class flare), it is reasonable to expect that stronger X-ray flares can induce disturbances important for satellite signals for a longer time period than in the considered case. This lasting depends on flare and atmospheric currently properties which need to be detailed investigated in future research.

Here, we note that the maximum obtained values of $P_{\mathrm{D}}$ (calculated for $\Theta_{0}=70^{\circ}$ which give the larger delay) in the unperturbed D-region are significantly less than $1 \mathrm{~cm}$ for all frequencies $\left(0.0014 \mathrm{~cm}\right.$ for $f_{1}, 8 \cdot 10^{-4} \mathrm{~cm}$ for $f_{2}$, and $7 \cdot 10^{-5}$ $\mathrm{cm}$ for $f_{3}$ ), which confirm the approximation that D-region can be ignored in modeling during quiet conditions.

\section{B. $P_{\mathrm{D}}$ Dependence on the X-Ray Flare Flux Maximum}

Calculations of the $P_{\mathrm{D}}$ dependence on the solar X-ray flare flux maximum $I_{\max }$ are performed using real observations of several tents X-ray flares presented in [11] and procedure described in Section III-B. Keeping in mind that ionospheric properties are space and time dependent, it is more adequate to use its fit parameters for estimation of $P_{\mathrm{D}}\left(I_{\max }\right)$. For this reason, we apply (7) to fit curves (given in [27]) of Wait's parameters determined in these observations.

In Fig. 3, one can see a significant influence of $I_{\max }$ on $P_{\mathrm{D}}$, especially in the case of the M-class X-ray flares $\left(I_{\max }>1 \cdot 10^{5}\right.$ $\mathrm{W} / \mathrm{m}^{-2}$ ), where $P_{\mathrm{D}}$ increases from several $\mathrm{cm}$ to about $2 \mathrm{~m}$ (for frequencies $f_{1}$ and $f_{2}$ ) going to larger incident angles $\Theta_{0}$. Table I gives estimated values of $P_{\mathrm{D}}$ at the flux maximum $I_{\max }$ of the $\mathrm{C} 1, \mathrm{C} 5, \mathrm{M} 1$, and M5 solar X-ray flares for the considered frequencies of the GNSS/SAR signals entering the D-region at angles between $0^{\circ}$ and $70^{\circ}$. Table II shows the $P_{\mathrm{D}}$ values estimated for real GPS signals received by four GPS
TABLE I

Estimated Values of $P_{\mathrm{D}}$ at The Flux Maximum of The C1, C5, M1, AND M5 SOlAR X-RAY Flares FOR $f_{1}, f_{2}$, AND $f_{3}$ FREQUENCIES OF THE GNSS/SAR SIGNALS ENTERING THE D-REGION AT ANGLES $0^{\circ}$ AND $70^{\circ}$

\begin{tabular}{ccccc}
\hline$I_{\max }$ & flare & \multicolumn{2}{c}{$P_{D}\left(\Theta=0^{\circ}\right)-P_{D}\left(\Theta=70^{\circ}\right)(\mathrm{cm})$} \\
$\left(\mathrm{W} / \mathrm{m}^{2}\right)$ & class & $f_{1}$ & $f_{2}$ & $f_{3}$ \\
\hline $10^{-6}$ & $\mathrm{C} 1$ & $0.3-0.8$ & $0.2-0.5$ & $0.01-0.04$ \\
$5 \cdot 10^{-6}$ & $\mathrm{C} 5$ & $2.3-7.0$ & $1.4-4.0$ & $0.1-0.3$ \\
$10^{-5}$ & M1 & $6.4-18.6$ & $3.7-10.8$ & $0.3-1.0$ \\
$5 \cdot 10^{-5}$ & M5 & $67.2-196.2$ & $39.0-113.9$ & $3.3-9.7$ \\
\hline
\end{tabular}

TABLE II

EXAMPlES OF $P_{D}$ FOR REAL OBSERVATIONS OF GPS Signals DETECTED By ReCEIVERS in NeW Zealand (Nelson, BlufF, Kaitaia, AND HICKS BAY, RESPECTIVELY) FOR FOUR X-RAY FlARES (THOMSon, Private Communication) Detected by GOES SatelLITES (www.ngdc.noaa.gov) AND SHOWN IN [11]

\begin{tabular}{|c|c|c|c|c|c|}
\hline $\begin{array}{l}\text { flare } \\
\text { class }\end{array}$ & $\begin{array}{c}\text { date } \\
t \text { (UT) }\end{array}$ & $\begin{array}{c}\beta\left(\mathrm{km}^{-1}\right) / \\
H^{\prime}(\mathrm{km})\end{array}$ & $\begin{array}{c}\text { GPS } \\
\text { satellite }\end{array}$ & $\begin{array}{l}\Theta_{0} \\
\left({ }^{\circ}\right)\end{array}$ & $\begin{array}{l}P_{D}(\mathrm{~cm}) \\
\left(f_{1} / f_{2}\right)\end{array}$ \\
\hline \multirow[t]{2}{*}{$\mathrm{C} 1$} & $1 / 12 / 97$ & $0.394 /$ & G27 & 15.302 & $0.45 / 0.26$ \\
\hline & $21: 43$ & 70.823 & G02 & 65.320 & $1.03 / 0.60$ \\
\hline \multirow[t]{2}{*}{$\mathrm{C} 5$} & $15 / 11 / 98$ & $0.443 /$ & G04 & 15.18 & $2.89 / 1.67$ \\
\hline & $22: 48$ & 68.312 & G08 & 52.67 & $4.59 / 2.67$ \\
\hline \multirow[t]{2}{*}{ M1 } & $3 / 11 / 97$ & $0.466 /$ & $\mathrm{G} 29$ & 14.303 & $8.57 / 4.97$ \\
\hline & $20: 16$ & 66.877 & $\mathrm{G} 22$ & 67.119 & $21.35 / 12.39$ \\
\hline \multirow[t]{2}{*}{ M2.4 } & $27 / 3 / 97$ & $0.465 /$ & G04 & 14.8 & $30.71 / 17.82$ \\
\hline & $22: 27$ & 64.094 & G13 & 65.161 & $70.66 / 41.00$ \\
\hline
\end{tabular}

stations during the maxima of four X-ray flares. $\Theta^{\circ}$ angles are obtained from positions of the considered GPS receivers and satellites at observational times using the procedure from [28] and data given in $\mathrm{ftp} / / /$ cddis.gsfc.nasa.gov/gnss/products/. $P_{\mathrm{D}}$ is calculated for the obtained angles $\Theta^{\circ}$ using the procedure given in Section III, and $\beta$ and $H^{\prime}$ parameters obtained by real VLF observations and presented in [11].

Finally, note that the nonnegligible contribution of the entire D-region in the GNSS/SAR positioning and remote sensing remains valid even in the case when calculations are applied only to the altitudes below $80 \mathrm{~km}$ where different models are in a better mutual agreement than at the upper part of the D-region [24]. For example, in this case, a delay of $1 \mathrm{~cm}$ can be reached for the $\mathrm{M}$ class flares at all considered angles $\Theta_{0}$ for frequencies $f_{1}$ and $f_{2}$, while the more intensive flares can produce such a delay also for frequency $f_{3}$.

\section{CONCLUSION}

A procedure for estimating the temporal evolution of the propagation delay during X-ray flares was presented. It is based on data collected in the low ionospheric monitoring by the VLF signals. The following are the main conclusions.

1) D-region delays during perturbations can be sufficiently large (several tens of minutes during solar X-ray flare) to cause pronounced impacts on the GNSS and SAR applications.

2) The GNSS/SAR signal incident angle affects the amount of $P_{\mathrm{D}}$ and duration time when the solar X-ray flare sufficiently alters the signal characteristics. 
3) The maximum flux of a solar X-ray flare significantly affects the D-region influence on the GNSS/SAR signals which increase with maximum radiation flux. That indicates that the very intensive X-ray flares may provide very serious errors in the corresponding measurements.

4) $P_{\mathrm{D}}$ decreases with the frequency of GNSS/SAR signals.

Because the SLM and MLM models do not include real observational data at the D-region heights as input parameters in the TEC calculation, they cannot see the $P_{D}$ increase during solar X-ray flares that mainly disturb the D-region (the E- and F-regions are significantly disturbed only by intensive X-ray flares). Although the increased $P_{D}$ is lower than the delay in the upper ionosphere, this effect is not negligible like in the case of quiet conditions and if not included can cause errors in GNSS and SAR applications. The methodology we propose can solve this problem by using VLF observations to quantify the $P_{D}$ value and correct the total delay in the ionosphere.

In GNSS applications, both for permanent receivers and single campaigns, we see GNSS data as a "continuous" function of time as the sampling time is of a few seconds. During X-ray flare, the propagation delay should be removed from the GNSS signal, within the time window corresponding to the X-ray flare duration. Instead, in interferometric SAR applications, SAR images which have been acquired during an X-ray flare are identified. Interferograms obtained by processing at least one of these SAR images should contain the D-region propagation delay, and, for them, the proposed methodology to compute the propagation delay should be applied.

\section{ACKNOWLEDGMENT}

The authors would like to thank N. Thomson for the help in preparation of our study. Requests for the VLF data used for analysis can be directed to the corresponding author.

The authors thank the Ministry of Education, Science and Technological Development of the Republic of Serbia for the support of this work within the projects 176001, 176002, 176004, III44002, III47007 and TR36020. This research was carried out in the framework of the OT4CLIMA Project which was funded by the Italian Ministry of Education, University and Research (D.D. 2261 del 6.9.2018, PON R\&I 2014-2020, and Fondo per lo Sviluppo e la Coesione), TD1403 and CA15211 COST Actions, and the VarSITI Project.

The data for this letter collected by GOES satellites are available at the NOAA's National Centers for Environmental information (http://satdat.ngdc.noaa.gov/sem/goes/data).

\section{REFERENCES}

[1] G. Wautelet, "Characterization of ionospheric irregularities and their influence on high-accuracy positioning with gps over mid-latitudes," Ph.D. dissertation, Univ. Liège, Liège, Belgium, 2013.

[2] G. Nico, R. Tome, J. Catalao, and P. M. A. Miranda, "On the use of the WRF model to mitigate tropospheric phase delay effects in SAR interferograms," IEEE Trans. Geosci. Remote Sens., vol. 49, no. 12, pp. 4970-4976, Dec. 2011.

[3] F. J. Meyer, "Performance requirements for ionospheric correction of low-frequency SAR data," IEEE Trans. Geosci. Remote Sens., vol. 49, no. 10, pp. 3694-3702, Oct. 2011.

[4] P. Mateus, P. M. A. Miranda, G. Nico, J. Catalão, P. Pinto, and R. Tomé, "Assimilating InSAR maps of water vapor to improve heavy rainfall forecasts: A case study with two successive storms," J. Geophys. Res., Atmos., vol. 123, pp. 3341-3355, Apr. 2018.
[5] F. Meyer, R. Bamler, N. Jakowski, and T. Fritz, "The potential of lowfrequency SAR systems for mapping ionospheric TEC distributions," IEEE Geosci. Remote Sens. Lett., vol. 3, no. 4, pp. 560-564, Oct. 2006.

[6] M. Bevis, S. Businger, T. A. Herring, C. Rocken, R. A. Anthes, and R. H. Ware, "GPS meteorology: Remote sensing of atmospheric water vapor using the global positioning system," J. Geophys. Res., vol. 97, pp. 15787-15801, Oct. 1992

[7] P. Benevides, J. Catalão, G. Nico, and P. M. A. Miranda, "4D wet refractivity estimation in the atmosphere using GNSS tomography initialized by radiosonde and AIRS measurements: Results from a 1 week intensive campaign," GPS Solutions, vol. 22, no. 4, p. 91, Jul. 2018.

[8] A. K. Singh, A. K. Singh, R. Singh, and R. Singh, "Solar flare induced D-region ionospheric perturbations evaluated from VLF measurements," Astrophys. Space Sci., vol. 350, no. 1, pp. 1-9, Mar. 2014.

[9] L. A. Hayes, P. T. Gallagher, J. McCauley, B. R. Dennis, J. Ireland, and A. Inglis, "Pulsations in the earth's lower ionosphere synchronized with solar flare emission," J. Geophys. Res., Space Phys., vol. 122, no. 10, pp. 9841-9847, Oct. 2017.

[10] A. Nina and V. Cadež, "Electron production by solar Ly- $\alpha$ line radiation in the ionospheric D-region," Adv. Space Res., vol. 54, no. 7, pp. 1276-1284, Oct. 2014.

[11] W. M. McRae and N. R. Thomson, "Solar flare induced ionospheric D-region enhancements from VLF phase and amplitude observations," J. Atmos. Solar-Terr. Phys., vol. 66, pp. 77-87, Jan. 2004.

[12] J. A. Klobuchar, "Design and characteristics of the GPS ionospheric time delay algorithm for single frequency users," in Proc. Position Location Navigat. Symp. (PLANS), New York, NY, USA, Nov. 1986, pp. 280-286.

[13] J. Zhao and C. Zhou, "On the optimal height of ionospheric shell for single-site TEC estimation," GPS Solutions, vol. 22, no. 2, p. 48 , Feb. 2018

[14] R. J. Daniell and L. Brown, PRISM: A Parameterized Real-Time Ionospheric Specification Model, Version 1.5. Newton, MA, USA: Computational Physics, 1995.

[15] B. Nava, P. Coïsson, and S. Radicella, "A new version of the NeQuick ionosphere electron density model," J. Atmos. Solar-Terr. Phys., vol. 70, no. 15 , pp. 1856-1862, Dec. 2008

[16] L. Scherliess, R. W. Schunk, J. J. Sojka, D. C. Thompson, and L. Zhu, "Utah State University global assimilation of ionospheric measurements Gauss-Markov Kalman filter model of the ionosphere: Model description and validation," J. Geophys. Res., Space Phys., vol. 111, Nov. 2006, Art. no. A11315.

[17] J. Maeda, T. Suzuki, M. Furuya, and K. Heki, "Imaging the midlatitude sporadic E plasma patches with a coordinated observation of spaceborne InSAR and GPS total electron content," Geophys. Res. Lett., vol. 43, no. 4, pp. 1419-1425, 2016.

[18] S. M. Stankov, R. Warnant, and K. Stegen, "Trans-ionospheric GPS signal delay gradients observed over mid-latitude Europe during the geomagnetic storms of October-November 2003," Adv. Space Res., vol. 43, no. 9, pp. 1314-1324, May 2009.

[19] D. P. Grubor, D. M. Šulić, and V. Žigman, "Classification of X-ray solar flares regarding their effects on the lower ionosphere electron density profile," Ann. Geophys., vol. 26, pp. 1731-1740, Jun. 2008

[20] J. A. Ferguson, Computer Programs for Assessment of Long-Wavelength Radio Communications, Version 2.0: User Guide. San Diego, CA, USA: Space and Naval Warfare Systems Center, 1998.

[21] J. R. Wait and K. P. Spies, "Characteristics Earth-ionosphere waveguide for VLF radio waves," Nat. Bureau Standards, Boulder, CO, USA, Tech. Note 300, 1964

[22] N. R. Thomson, "Experimental daytime VLF ionospheric parameters," J. Atmos. Terr. Phys., vol. 55, pp. 173-184, Feb. 1993.

[23] A. Kumar and S. Kumar, "Solar flare effects on D-region ionosphere using VLF measurements during low- and high-solar activity phases of solar cycle 24," Earth Planets Space, vol. 70, no. 1, Feb. 2018, Art. no. 29

[24] E. D. Schmitter, "Modeling solar flare induced lower ionosphere changes using VLF/LF transmitter amplitude and phase observations at a midlatitude site," Ann. Geophys., vol. 31, no. 4, pp. 765-773, Apr. 2013.

[25] A. Ishimaru, Electromagnetic Wave Propagation, Radiation, and Scattering. Hoboken, NJ, USA: Wiley, 2017.

[26] M. Radovanović, "Investigation of solar influence on the terrestria processes: Activities in Serbia," J. Geograph. Inst. Cvijic, vol. 68, no. 1, pp. 149-155, Apr. 2018.

[27] M. Todorović Drakul, V. M. Čadež, J. Bajčetić, D. B. L. C. Popović, and A. Nina, "Behaviour of electron content in the ionospheric D-region during solar X-ray flares," Serbian Astronomical J., vol. 193, pp. 11-18, Dec. 2016.

[28] G. Xu, GPS: Theory, Algorithms and Applications. Berlin, Germany: Springer, 2007. 


\title{
GNSS and SAR Signal Delay in Perturbed Ionospheric D-Region During Solar X-Ray Flares
}

\author{
Aleksandra Nina ${ }^{\circledR}$, Giovanni Nico ${ }^{\circledR}$, Senior Member, IEEE, Oleg Odalović, Vladimir M. Čadež, \\ Miljana Todorović Drakul, Milan Radovanović, and Luka Č. Popović
}

\begin{abstract}
We investigate the influence of the perturbed (by a solar X-ray flare) ionospheric D-region on the global navigation satellite systems (GNSS) and synthetic aperture radar (SAR) signals. We calculate a signal delay in the D-region based on the low ionospheric monitoring by very-low-frequency (VLF) radio waves. The results show that the ionospheric delay in the perturbed D-region can be important and, therefore, should be taken into account in modeling the ionospheric influence on the GNSS and SAR signal propagation and in calculations relevant for space geodesy. This conclusion is significant because numerous existing models ignore the impact of this ionospheric part on the GNSS and SAR signals due to its small electron density which is true only in quiet conditions and can result in significant errors in space geodesy during intensive ionospheric disturbances.
\end{abstract}

Index Terms-Global navigation satellite systems (GNSS), ionosphere, synthetic aperture radar (SAR) interferometry (InSAR), very-low-frequency (VLF) radio signals.

\section{INTRODUCTION}

$\mathbf{N}$ OWADAYS spaceborne measurements of positioning, displacements, navigation, and timing play an important and critical role in telecommunications, geodesy, all forms of transportation and other human activities. These measurements are primarily provided by the global navigation

Manuscript received September 20, 2018; revised February 6, 2019 and July 15, 2019; accepted September 4, 2019. This work was supported by the Ministry of Education, Science and Technological Development of the Republic of Serbia under Projects 176001, 176002, 176004, III44002, III47007, and TR36020, and the OT4CLIMA Project which was funded by the Italian Ministry of Education, University and Research (D.D. 2261 del 6.9.2018, PON R\&I 2014-2020, and Fondo per lo Sviluppo e la Coesione). (Corresponding author: Aleksandra Nina.)

A. Nina is with the Institute of Physics Belgrade, University of Belgrade, 11080 Belgrade, Serbia (e-mail: sandrast@ipb.ac.rs).

G. Nico is with the Istituto per le Applicazioni del Calcolo (IAC), Consiglio Nazionale delle Ricerche (CNR), 70126 Bari, Italy, and also with the Department of Cartography and Geoinformatics, Institute of Earth Sciences, Saint Petersburg State University (SPSU), 199034 Saint Petersburg, Russia (e-mail: g.nico@ba.iac.cnr.it).

O. Odalović and M. Todorović Drakul are with the Department of Geodesy and Geoinformatics, Faculty of Civil Engineering, University of Belgrade, 11000 Belgrade, Serbia.

V. M. Čadež is with the Astronomical Observatory, 11060 Belgrade, Serbia. M. Radovanović is with the Geographical Institute "Jovan Cvijić" SASA, 11000 Belgrade, Serbia, and also with the Institute of Sports, Tourism and Service, South Ural State University, 454080 Chelyabinsk, Russia.

L. C. Popović is with the Astronomical Observatory, 11060 Belgrade, Serbia, and also with the Faculty of Science, University of Banja Luka, 78000 Banja Luka, Bosnia and Herzegovina.

Color versions of one or more of the figures in this letter are available online at http://ieeexplore.iee.org.

Digital Object Identifier 10.1109/LGRS.2019.2941643 satellite systems (GNSS) and synthetic aperture radar (SAR) interferometry (InSAR). In the case of GNSS signals, the accuracy of positioning depends on both the errors in measurements and the way of modeling different influences on signal propagation. There are many sources causing these errors and their modeling indicates various effects on cumulative error. The methodology presented in this article is intended to be applied to single-frequency GNSS receivers. For these receivers, one should know the precise satellite orbits, satellite clock errors and hardware delay, receiver hardware, ionospheric and tropospheric delay, multipath and phase center variation which delays are $2.5-5 \mathrm{~cm}$, up to $2 \mathrm{~cm}$, up to $3 \mathrm{~m}$, 2.3-5 m, 6 and up to $1 \mathrm{~cm}$, respectively [1]. This indicates that sources contributing errors of already $1 \mathrm{~cm}$ have to be included in calculations of the GNSS positioning. As far as InSAR is concerned, the measurement of displacements (landslides, earthquakes, glaciers, and infrastructures) with a subcentimetric precision requires the knowledge of precise orbits, high-resolution digital elevation models and good modeling of propagation effects in the atmosphere, including both troposphere and ionosphere [2], [3]. Recently, the potential of InSAR to provide maps of tropospheric and ionospheric delays with high spatial resolution, even for low temporal sampling, was proven [4], [5]. In particular, SAR meteorology is complementing the techniques of GNSS meteorology [6] and GNSS tomography (see [7]) to measure the atmospheric water vapor.

The ionosphere can be a significant source of delay for the GNSS and SAR signals. This deviation is caused by free electrons whose density is significantly larger in the upper ionosphere. For this reason, modeling of the ionospheric influences on satellite signals is often based on the analyses for altitudes above $90 \mathrm{~km}$. Also, even a model includes the D-region $(60-90 \mathrm{~km})$ in calculations, input parameters obtained in observations are relevant for one or a few altitudes above this layer, and a part of the total electron content (TEC) in the D-region is calculated using extrapolation with the unique expressions for all conditions including cases when perturbations are dominant or only exist below $90 \mathrm{~km}$. However, this limitation/extrapolation (applicable to the quiet conditions) opens a question: Can the perturbed D-region sufficiently affect the GNSS and SAR signals so that the inclusion of the perturbed D-region observation data in models becomes necessary for measurements? 
Lack of studies directed to research of these approximations motivated us to study the influence of the perturbed D-region on GNSS and SAR signals. We consider perturbations induced by a solar X-ray flare which is one of the phenomena whose occurrence primarily disturbs the low ionosphere and can cause an increase of the electron density by one or two orders of magnitude in the D-region [8], [9].

There are two goals of this study. First, we investigate the properties of the time evolution of the ionospheric delay in the $\mathrm{D}$-region during a solar X-ray flare. The fact that X-ray flares induce similar properties of the D-region electron density time and space distributions (see [10]) which are required for delay calculations allows us to use one solar X-ray flare as an example (appeared on May 1, 2013). Second, we analyze the D-region delay dependencies from the $\mathrm{X}$ radiation flux maximum using observations given in [11].

\section{IONOSPHERIC DELAY: DifFERENT CONTRIBUtions}

The ionospheric influence on the GNSS/SAR signal deviation can be described by the ionospheric delay $P_{\mathrm{I}}=\alpha_{\mathrm{I}} \mathrm{TEC} / f^{2}$ where $\alpha_{\mathrm{I}}=40.3 \mathrm{~m}^{3} \mathrm{~s}^{-2}$, and TEC $=\int_{l_{\mathrm{I}}} N_{\mathrm{e}} d l_{\mathrm{I}}$ is the TEC (in $\mathrm{m}^{-2}$ ) along the ray path of length $l_{\mathrm{I}}$ in the ionosphere with the electron density $N_{\mathrm{e}}$.

Determination of TEC is not a simple task because of a nonstationary and complex electron density spatial distribution. The F-region has the most important influence on $P_{\mathrm{I}}$ and the contribution of other layers decrease with altitude. For this reason, TEC is generally calculated by single-layer models (SLM) which assume the entire effect localized to some fixed height in the F-region [12], [13], or multiple-layer models (MLM) which used observation data at the ionospheric E- and F-region height even the D-region contribution is included in total TEC [14]- [16]. However, the ionosphere is under permanent influences of ionizing radiation from the outer space and terrestrial layers which can significantly increase the electron density and result in the increase of the ionospheric influence on the GNSS/SAR signals [17], [18]. The importance of these effects varies with altitude which can indicate a need for additional measurements modeling.

\section{Modeling of the D-Region Delay}

Like for the entire ionosphere, the D-region delay can be expressed as

$$
P_{\mathrm{D}}=\alpha_{\mathrm{I}} \mathrm{TEC}_{\mathrm{D}} / f^{2} .
$$

The $\mathrm{TEC}_{\mathrm{D}}=\int_{l_{\mathrm{D}}} N_{\mathrm{e}} d l_{\mathrm{D}}$ for the signal propagation path $l_{\mathrm{D}}$ in the D-region can be obtained from models of the electron density space and time distributions $N_{\mathrm{e}}$, and the considered signal propagation path.

\section{A. D-Region Electron Density}

Determination of the electron density is a very complex issue for both in situ measurements by rockets and remote sensing by radio waves. The reasons are the absence of sudden event detections as the measurements are not continuous in time, medium perturbations caused by the moving rocket which creates errors in obtained quantities and lack of knowledge of numerous parameters which are usually unavailable for the considered location during the observation. Nowadays, the radio signals are most frequently utilized in the low ionospheric monitoring which provides necessary data for various models to compute the D-region plasma parameters during perturbations induced by different sudden events.

In this letter, determination of the electron density during an $\mathrm{X}$-ray flare is achieved by a procedure [19] based on matching changes of the observed very-low-frequency (VLF) signal (its amplitude $\Delta A$ and phase $\Delta P$ ) with corresponding values resulting from simulations of the VLF signal propagation using the long-wave propagation capability (LWPC) numerical model developed by the Naval Ocean Systems Center, San Diego, USA [20]. The simulated values of amplitude and phase are obtained by varying two independent parameters, the "sharpness" $\beta$ and the signal reflection height $H^{\prime}$, which characterize Wait's model of the ionosphere [21]. Finally, the values of parameters that yield the best fit of simulated values with experimental data are used to determine the electron density according to the equation for the horizontal uniform ionosphere [22]

$$
N_{\mathrm{e}}(h, t)=1.43 \cdot 10^{13} e^{-\beta(t) H^{\prime}(t)} e^{(\beta(t)-0.15) h}
$$

which is commonly used for the D-region electron density modeling in numerous papers [9], [10], [23]. Here, $N_{\mathrm{e}}, \beta$ and $H^{\prime}$ are given in $\mathrm{m}^{-3}, \mathrm{~km}^{-1}$, and $\mathrm{km}$, respectively.

It is worth noting that the form of (2) depends on the ionosphere model. Differences among models are more pronounced both at higher D-region altitudes and for more intense solar X-ray flares [24]. For these reasons, we consider flares of classes lower than M5, and compare results for the entire D-region $(60-90 \mathrm{~km})$ with those for a reduced altitude range below $80 \mathrm{~km}$, where the expected influence of model selection is less pronounced.

\section{B. GNSS/SAR Signal Propagation Path in the D-Region}

The D-region of thickness $H_{\mathrm{D}}$ is modeled as a set of $N_{\mathrm{D}}$ horizontally uniform layers of thickness $\delta=H_{\mathrm{D}} / N_{\mathrm{D}}$. The GNSS/SAR signal refracts at the $i$ th layer according to the refractive index $n_{\mathrm{i}}$ [25]

$$
n_{\mathrm{i}}=\sqrt{1-f_{\mathrm{pi}}^{2} / f^{2}}
$$

where $f$ is the signal frequency, $f_{\mathrm{pi}}=8.98\left(N_{\mathrm{ei}}\right)^{1 / 2}$ (in $\mathrm{Hz}$ ) is the electron plasma frequency at the $i$ th horizontal uniform layer with electron density $N_{\mathrm{ei}}$ (in $\mathrm{m}^{-3}$ ) centered around the height $h_{i}$ related to the middle of the $i$-th layer. Here, we notice that the collision frequency is ignored because calculations showed its negligible influence on the refractive index. Refraction on the boundaries can be described by Snell's law [25]

$$
n_{\mathrm{i}} \sin \left(\Theta_{\mathrm{i}}\right)=n_{\mathrm{i}-1} \sin \left(\Theta_{\mathrm{i}-1}\right)=n_{0} \sin \left(\Theta_{0}\right)
$$

where $i=1,2, \ldots, \mathrm{N}_{\mathrm{D}}+1$ and $\Theta_{i}$ is the wave propagation angle in the $i$ th layer. According to (4) in individual layer $i$ of thickness $\delta H_{\mathrm{D}}$, the wave path length $l_{\mathrm{i}}=\delta H_{\mathrm{D}} / \cos \left(\Theta_{\mathrm{i}}\right)$ is

$$
l_{i}=n_{\mathrm{i}} \delta H_{\mathrm{D}}\left[n_{\mathrm{i}}{ }^{2}-\left(n_{0} \sin \left(\Theta_{0}\right)\right)^{2}\right]^{-0.5}
$$




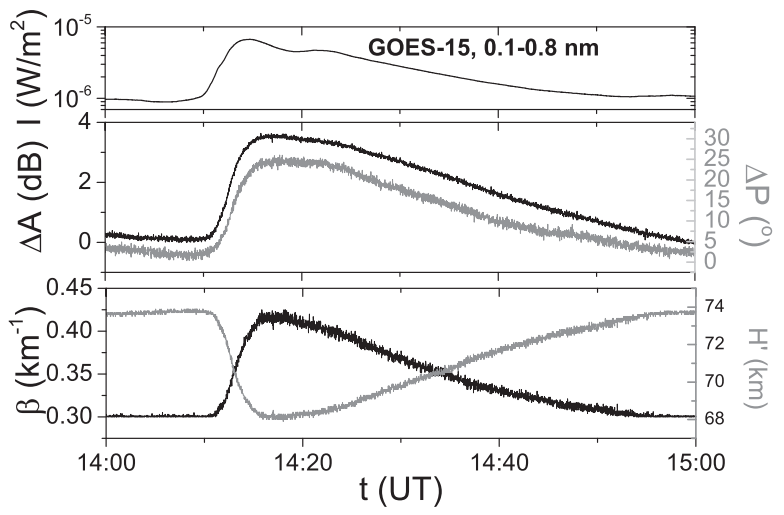

Fig. 1. (Top) Time dependencies of the X-ray radiation flux recorded by the GOES-15 satellite. (Middle) Signal amplitude and phase recorded in Serbia. (Bottom) Wait's parameters calculated by the procedure given in Section III.

where $\Theta_{0}$ and $n_{0}$ are the incident angle of the GNSS/SAR signal in the D-region and refractive index of the upper layer.

\section{C. $P_{D}$ Modeling}

In this study, we derived expression for calculation of the D-region delay using the above explained model for the electron density calculation (Section III-A) and presented procedure for the GNSS/SAR signal path determination (Section III-B). From (5) and $\mathrm{TEC}_{\mathrm{D}}=\sum_{i=1}^{N_{\mathrm{D}}} N_{\mathrm{ei}} \mathrm{l}_{\mathrm{i}}$, we first obtain

$$
\mathrm{TEC}_{\mathrm{D}}=\delta H_{\mathrm{D}} \sum_{i=1}^{N_{\mathrm{D}}} N_{\mathrm{ei}} n_{\mathrm{i}}\left[n_{\mathrm{i}}{ }^{2}-\left(n_{0} \sin \left(\Theta_{0}\right)\right)^{2}\right]^{-0.5} .
$$

Finally, knowing the incident angle of the GNSS/SAR signal in the D-region, $\Theta_{0}$, and altitude distributions of the electron density $N_{\mathrm{e}}$ necessary for calculation of the refractive index according (3), we express $P_{\mathrm{D}}$ from (1) and (6)

$$
P_{\mathrm{D}}=\frac{C \delta H_{D}}{f^{2}} \sum_{i=1}^{N_{\mathrm{D}}} N_{\mathrm{ei}} n_{\mathrm{i}}\left[n_{\mathrm{i}}^{2}-\left(n_{0} \sin \left(\Theta_{0}\right)\right)^{2}\right]^{-0.5}
$$

for the modeled D-region by $N_{\mathrm{D}}$ uniform layers with depth $\delta H_{\mathrm{D}}$. We notice that, in the cases of local perturbations, the used approximation of horizontal uniform layers is not possible and more data sources need to be included in modeling.

\section{OBSERVATIONS AND DATA}

The first part of our analysis is directed to ionospheric perturbations induced by the solar X-ray flare of May 1, 2013. The increase of photon flux recorded by the National Oceanic and Atmospheric Administration (NOAA) satellite GOES-15, and its time evolution within the wavelengths range $0.1-0.8 \mathrm{~nm}$ is shown in Fig. 1 (top).

Data for the low ionospheric modeling were obtained using the 23.4-kHz VLF signal emitted by the DHO transmitter located in Rhauderfehn (Germany) and received at the Institute of Physics in Belgrade (Serbia). This transmitter was chosen because it provides a relatively short signal propagation path as well as the most suitable signal characteristics for the location of the receiver [26]. The ionospheric perturbations

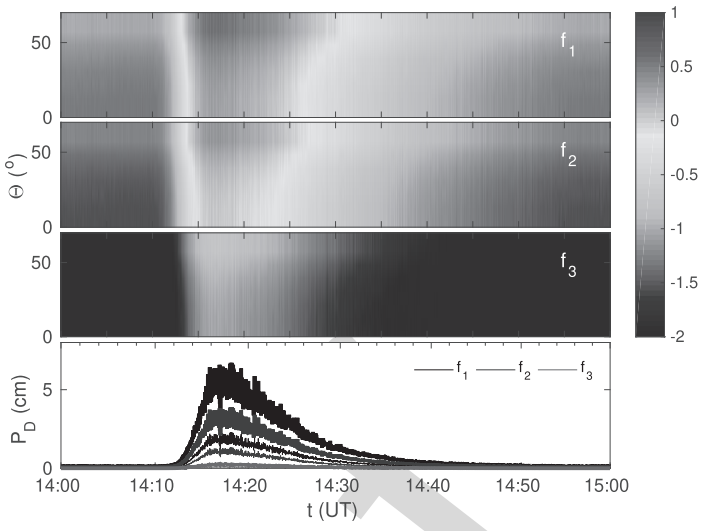

Fig. 2. (Top three panels) Time evolutions of $\log \left(P_{\mathrm{D}} / 1 \mathrm{~cm}\right)$ for incident angles $\Theta_{0}$ in the range $0^{\circ}-70^{\circ}$ and GNSS/SAR frequencies $f_{1}=1.20000$ $\mathrm{GHz}, f_{2}=1.57542 \mathrm{GHz}$, and $f_{3}=5.40500 \mathrm{GHz}$. (Bottom panel) Time evolutions of $P_{\mathrm{D}}$ for incident angles of $0^{\circ}$ and $70^{\circ}$ for the considered frequencies.

were detected as the amplitude $\Delta A$ and phase $\Delta P$ variations of the considered VLF signal Fig. 1 (middle) recorded by the Absolute Phase and Amplitude Logger (AbsPAL) VLF receiver. The Wait's parameters $\beta$ and $H^{\prime}$, as shown in Fig. 1 (bottom), are obtained by applying the numerical procedure described in Section III to these data. A good agreement was found with results presented in [10], [11], and [19]. Their implementation in analytical expressions also given in Section III provides the time evolution of the D-region time delay.

In the second part of this study, we used data from literature which are also obtained from the low ionospheric observations by the VLF signals [11].

\section{RESULTS AND DISCUSSION}

The procedure given in Section III is applied to the data recorded by the Belgrade very low/low frequency (VLF/LF) receiver station: at the time of solar X-ray flare of May 1, 2013 (the first part of this study) and at the time of maximum flux of solar X-ray flares considered in [11]. In both parts, we considered three GNSS/SAR frequencies $f_{1}=1.2 \mathrm{GHz}$ (GNSS, ALOS-2 or the future NISAR mission), $f_{2}=1.57542 \mathrm{GHz}$ (GNSS), and $f_{3}=5.4 \mathrm{GHz}$ (Sentinel-1 and Radarsat-2) with incident angles $\Theta_{0}$ between $0^{\circ}$ and $70^{\circ}$ (relevant for the satellite positioning).

\section{A. Time Evolution of $P_{\mathrm{D}}$-Example of Particular Event}

A visualization of influences of the GNSS/SAR signal incident angle $\Theta_{0}$ and the signal frequency $f$ to the D-region delay $P_{\mathrm{D}}$ during the considered event is shown in Fig. 2. As one can see the D-region influence increases with incident angle due to increasing of signal propagation path within the D-region and, consequently, $\mathrm{TEC}_{\mathrm{D}}$ (see Section III). It is lowest for the signal with frequency $f_{3}$ which is the highest considered frequency. The decrease of $P_{\mathrm{D}}$ with frequency can be explained by (7), which indicates that $P_{\mathrm{D}} \sim f^{-2}$. The other two frequencies show very similar time evolutions of $P_{\mathrm{D}}$.

The time evolutions of $P_{\mathrm{D}}$, as shown in Fig. 2, illustrate that the changes of $P_{\mathrm{D}}$ reach more than two orders of magnitude 


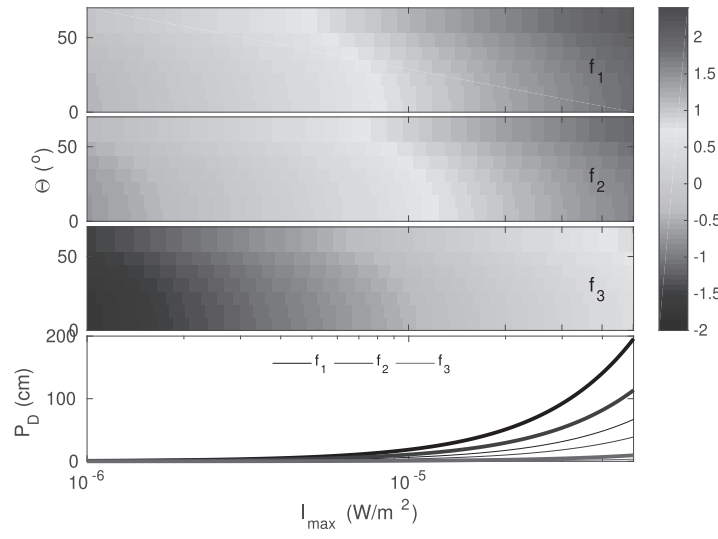

Fig. 3. (Top three panels) $P_{\mathrm{D}}$ dependence on $I_{\max }$ for incident angles $\Theta_{0}$ in the range $0^{\circ}-70^{\circ}$ and GNSS/SAR frequencies $f_{1}=1.20000 \mathrm{GHz}$, $f_{2}=1.57542 \mathrm{GHz}$, and $f_{3}=5.40500 \mathrm{GHz}$. (Bottom panel) Time evolutions of $P_{\mathrm{D}}$ for incident angles of $0^{\circ}$ and $70^{\circ}$ for the considered frequencies.

larger values when the considered flare event is present. These values reach a few centimeters which, keeping in mind that errors of about $1 \mathrm{~cm}$ are already considered nonnegligible in modeling, clearly indicates the significance of the role the perturbed D-region plays in accuracy of the GNSS/SAR measurements during a solar X-ray flare activity. In this figure, it is visible that time period with $P_{\mathrm{D}}>1 \mathrm{~cm}$ depends on the considered frequency $f$ (it decrease with frequency because decreasing of $P_{\mathrm{D}}$ ) and angle $\Theta_{0}$ (it increase with $\Theta_{0}$ due to increasing of $P_{\mathrm{D}}$ in time), and that this period can take several tens of minutes. Keeping in mind that the considered X-ray solar flare is not so strong (the C-class flare), it is reasonable to expect that stronger X-ray flares can induce disturbances important for satellite signals for a longer time period than in the considered case. This lasting depends on flare and atmospheric currently properties which need to be detailed investigated in future research.

Here, we note that the maximum obtained values of $P_{\mathrm{D}}$ (calculated for $\Theta_{0}=70^{\circ}$ which give the larger delay) in the unperturbed D-region are significantly less than $1 \mathrm{~cm}$ for all frequencies $\left(0.0014 \mathrm{~cm}\right.$ for $f_{1}, 8 \cdot 10^{-4} \mathrm{~cm}$ for $f_{2}$, and $7 \cdot 10^{-5}$ $\mathrm{cm}$ for $f_{3}$ ), which confirm the approximation that D-region can be ignored in modeling during quiet conditions.

\section{B. $P_{\mathrm{D}}$ Dependence on the X-Ray Flare Flux Maximum}

Calculations of the $P_{\mathrm{D}}$ dependence on the solar X-ray flare flux maximum $I_{\max }$ are performed using real observations of several tents X-ray flares presented in [11] and procedure described in Section III-B. Keeping in mind that ionospheric properties are space and time dependent, it is more adequate to use its fit parameters for estimation of $P_{\mathrm{D}}\left(I_{\max }\right)$. For this reason, we apply (7) to fit curves (given in [27]) of Wait's parameters determined in these observations.

In Fig. 3, one can see a significant influence of $I_{\max }$ on $P_{\mathrm{D}}$, especially in the case of the M-class X-ray flares $\left(I_{\max }>1 \cdot 10^{5}\right.$ $\mathrm{W} / \mathrm{m}^{-2}$ ), where $P_{\mathrm{D}}$ increases from several $\mathrm{cm}$ to about $2 \mathrm{~m}$ (for frequencies $f_{1}$ and $f_{2}$ ) going to larger incident angles $\Theta_{0}$. Table I gives estimated values of $P_{\mathrm{D}}$ at the flux maximum $I_{\max }$ of the $\mathrm{C} 1, \mathrm{C} 5, \mathrm{M} 1$, and M5 solar X-ray flares for the considered frequencies of the GNSS/SAR signals entering the D-region at angles between $0^{\circ}$ and $70^{\circ}$. Table II shows the $P_{\mathrm{D}}$ values estimated for real GPS signals received by four GPS
TABLE I

Estimated Values of $P_{\mathrm{D}}$ at the Flux Maximum of the C1, C5, M1, AND M5 SOLAR X-RAY Flares For $f_{1}, f_{2}$, AND $f_{3}$ FREQUENCIES OF THE GNSS/SAR SIGNALS ENTERING THE D-REGION AT ANGLES $0^{\circ}$ AND $70^{\circ}$

\begin{tabular}{ccccc}
\hline$I_{\max }$ & flare & \multicolumn{2}{c}{$P_{D}\left(\Theta=0^{\circ}\right)-P_{D}\left(\Theta=70^{\circ}\right)(\mathrm{cm})$} \\
$\left(\mathrm{W} / \mathrm{m}^{2}\right)$ & class & $f_{1}$ & $f_{2}$ & $f_{3}$ \\
\hline $10^{-6}$ & C1 & $0.3-0.8$ & $0.2-0.5$ & $0.01-0.04$ \\
$5 \cdot 10^{-6}$ & C5 & $2.3-7.0$ & $1.4-4.0$ & $0.1-0.3$ \\
$10^{-5}$ & M1 & $6.4-18.6$ & $3.7-10.8$ & $0.3-1.0$ \\
$5 \cdot 10^{-5}$ & M5 & $67.2-196.2$ & $39.0-113.9$ & $3.3-9.7$ \\
\hline
\end{tabular}

TABLE II

Examples of $P_{D}$ FOR Real ObSERVATIONS OF GPS Signals Detected By ReCeivers in New Zealand (Nelson, Bluff, Kaitaia, and Hicks BAy, RESPECTIVELY) FOR FOUR X-RAY FlaRES (THOMSon, Private Communication) Detected by GOES SatelLITES (www.ngdc.noaa.gov) AND SHOWN IN [11]

\begin{tabular}{cccccc}
\hline $\begin{array}{c}\text { flare } \\
\text { class }\end{array}$ & $\begin{array}{c}\text { date } \\
\text { C1 }\end{array}$ & $\beta\left(\mathrm{km}^{-1}\right) /$ & $\begin{array}{c}\text { GPS } \\
H^{\prime}(\mathrm{km})\end{array}$ & $\begin{array}{c}\Theta_{0} \\
\text { satellite }\end{array}$ & $\begin{array}{c}P_{D}(\mathrm{~cm}) \\
\left(f_{1} / f_{2}\right)\end{array}$ \\
\hline C1 & $1 / 12 / 97$ & $0.394 /$ & G27 & 15.302 & $0.45 / 0.26$ \\
& $21: 43$ & 70.823 & G02 & 65.320 & $1.03 / 0.60$ \\
C5 & $15 / 11 / 98$ & $0.443 /$ & G04 & 15.18 & $2.89 / 1.67$ \\
& $22: 48$ & 68.312 & G08 & 52.67 & $4.59 / 2.67$ \\
M1 & $3 / 11 / 97$ & $0.466 /$ & G29 & 14.303 & $8.57 / 4.97$ \\
& $20: 16$ & 66.877 & G22 & 67.119 & $21.35 / 12.39$ \\
M2.4 & $27 / 3 / 97$ & $0.465 /$ & G04 & 14.8 & $30.71 / 17.82$ \\
& $22: 27$ & 64.094 & G13 & 65.161 & $70.66 / 41.00$ \\
\hline
\end{tabular}

stations during the maxima of four X-ray flares. $\Theta^{\circ}$ angles are obtained from positions of the considered GPS receivers and satellites at observational times using the procedure from [28] and data given in $\mathrm{ftp} / / /$ cddis.gsfc.nasa.gov/gnss/products/. $P_{\mathrm{D}}$ is calculated for the obtained angles $\Theta^{\circ}$ using the procedure given in Section III, and $\beta$ and $H^{\prime}$ parameters obtained by real VLF observations and presented in [11].

Finally, note that the nonnegligible contribution of the entire D-region in the GNSS/SAR positioning and remote sensing remains valid even in the case when calculations are applied only to the altitudes below $80 \mathrm{~km}$ where different models are in a better mutual agreement than at the upper part of the D-region [24]. For example, in this case, a delay of $1 \mathrm{~cm}$ can be reached for the $\mathrm{M}$ class flares at all considered angles $\Theta_{0}$ for frequencies $f_{1}$ and $f_{2}$, while the more intensive flares can produce such a delay also for frequency $f_{3}$.

\section{CONCLUSION}

A procedure for estimating the temporal evolution of the propagation delay during X-ray flares was presented. It is based on data collected in the low ionospheric monitoring by the VLF signals. The following are the main conclusions.

1) D-region delays during perturbations can be sufficiently large (several tens of minutes during solar X-ray flare) to cause pronounced impacts on the GNSS and SAR applications.

2) The GNSS/SAR signal incident angle affects the amount of $P_{\mathrm{D}}$ and duration time when the solar X-ray flare sufficiently alters the signal characteristics. 
3) The maximum flux of a solar X-ray flare significantly affects the D-region influence on the GNSS/SAR signals which increase with maximum radiation flux. That indicates that the very intensive X-ray flares may provide very serious errors in the corresponding measurements.

4) $P_{\mathrm{D}}$ decreases with the frequency of GNSS/SAR signals.

Because the SLM and MLM models do not include real observational data at the D-region heights as input parameters in the TEC calculation, they cannot see the $P_{D}$ increase during solar X-ray flares that mainly disturb the D-region (the E- and F-regions are significantly disturbed only by intensive X-ray flares). Although the increased $P_{D}$ is lower than the delay in the upper ionosphere, this effect is not negligible like in the case of quiet conditions and if not included can cause errors in GNSS and SAR applications. The methodology we propose can solve this problem by using VLF observations to quantify the $P_{D}$ value and correct the total delay in the ionosphere.

In GNSS applications, both for permanent receivers and single campaigns, we see GNSS data as a "continuous" function of time as the sampling time is of a few seconds. During X-ray flare, the propagation delay should be removed from the GNSS signal, within the time window corresponding to the X-ray flare duration. Instead, in interferometric SAR applications, SAR images which have been acquired during an X-ray flare are identified. Interferograms obtained by processing at least one of these SAR images should contain the D-region propagation delay, and, for them, the proposed methodology to compute the propagation delay should be applied.

\section{ACKNOWLEDGMENT}

The authors would like to thank N. Thomson for the help in preparation of our study. Requests for the VLF data used for analysis can be directed to the corresponding author.

The authors thank the Ministry of Education, Science and Technological Development of the Republic of Serbia for the support of this work within the projects 176001, 176002, 176004, III44002, III47007 and TR36020. This research was carried out in the framework of the OT4CLIMA Project which was funded by the Italian Ministry of Education, University and Research (D.D. 2261 del 6.9.2018, PON R\&I 2014-2020, and Fondo per lo Sviluppo e la Coesione), TD1403 and CA15211 COST Actions, and the VarSITI Project.

The data for this letter collected by GOES satellites are available at the NOAA's National Centers for Environmental information (http://satdat.ngdc.noaa.gov/sem/goes/data).

\section{REFERENCES}

[1] G. Wautelet, "Characterization of ionospheric irregularities and their influence on high-accuracy positioning with gps over mid-latitudes," Ph.D. dissertation, Univ. Liège, Liège, Belgium, 2013.

[2] G. Nico, R. Tome, J. Catalao, and P. M. A. Miranda, "On the use of the WRF model to mitigate tropospheric phase delay effects in SAR interferograms," IEEE Trans. Geosci. Remote Sens., vol. 49, no. 12, pp. 4970-4976, Dec. 2011.

[3] F. J. Meyer, "Performance requirements for ionospheric correction of low-frequency SAR data," IEEE Trans. Geosci. Remote Sens., vol. 49, no. 10, pp. 3694-3702, Oct. 2011.

[4] P. Mateus, P. M. A. Miranda, G. Nico, J. Catalão, P. Pinto, and R. Tomé, "Assimilating InSAR maps of water vapor to improve heavy rainfall forecasts: A case study with two successive storms," J. Geophys. Res., Atmos., vol. 123, pp. 3341-3355, Apr. 2018.
[5] F. Meyer, R. Bamler, N. Jakowski, and T. Fritz, "The potential of lowfrequency SAR systems for mapping ionospheric TEC distributions," IEEE Geosci. Remote Sens. Lett., vol. 3, no. 4, pp. 560-564, Oct. 2006.

[6] M. Bevis, S. Businger, T. A. Herring, C. Rocken, R. A. Anthes, and R. H. Ware, "GPS meteorology: Remote sensing of atmospheric water vapor using the global positioning system," J. Geophys. Res., vol. 97, pp. 15787-15801, Oct. 1992

[7] P. Benevides, J. Catalão, G. Nico, and P. M. A. Miranda, "4D wet refractivity estimation in the atmosphere using GNSS tomography initialized by radiosonde and AIRS measurements: Results from a 1 week intensive campaign," GPS Solutions, vol. 22, no. 4, p. 91, Jul. 2018.

[8] A. K. Singh, A. K. Singh, R. Singh, and R. Singh, "Solar flare induced D-region ionospheric perturbations evaluated from VLF measurements," Astrophys. Space Sci., vol. 350, no. 1, pp. 1-9, Mar. 2014.

[9] L. A. Hayes, P. T. Gallagher, J. McCauley, B. R. Dennis, J. Ireland, and A. Inglis, "Pulsations in the earth's lower ionosphere synchronized with solar flare emission," J. Geophys. Res., Space Phys., vol. 122, no. 10, pp. 9841-9847, Oct. 2017.

[10] A. Nina and V. Cadež, "Electron production by solar Ly- $\alpha$ line radiation in the ionospheric D-region," Adv. Space Res., vol. 54, no. 7, pp. 1276-1284, Oct. 2014.

[11] W. M. McRae and N. R. Thomson, "Solar flare induced ionospheric D-region enhancements from VLF phase and amplitude observations," J. Atmos. Solar-Terr. Phys., vol. 66, pp. 77-87, Jan. 2004.

[12] J. A. Klobuchar, "Design and characteristics of the GPS ionospheric time delay algorithm for single frequency users," in Proc. Position Location Navigat. Symp. (PLANS), New York, NY, USA, Nov. 1986, pp. 280-286.

[13] J. Zhao and C. Zhou, "On the optimal height of ionospheric shell for single-site TEC estimation," GPS Solutions, vol. 22, no. 2, p. 48 , Feb. 2018

[14] R. J. Daniell and L. Brown, PRISM: A Parameterized Real-Time Ionospheric Specification Model, Version 1.5. Newton, MA, USA: Computational Physics, 1995.

[15] B. Nava, P. Coïsson, and S. Radicella, "A new version of the NeQuick ionosphere electron density model," J. Atmos. Solar-Terr. Phys., vol. 70, no. 15 , pp. 1856-1862, Dec. 2008

[16] L. Scherliess, R. W. Schunk, J. J. Sojka, D. C. Thompson, and L. Zhu, "Utah State University global assimilation of ionospheric measurements Gauss-Markov Kalman filter model of the ionosphere: Model description and validation," J. Geophys. Res., Space Phys., vol. 111, Nov. 2006, Art. no. A11315.

[17] J. Maeda, T. Suzuki, M. Furuya, and K. Heki, "Imaging the midlatitude sporadic E plasma patches with a coordinated observation of spaceborne InSAR and GPS total electron content," Geophys. Res. Lett., vol. 43, no. 4, pp. 1419-1425, 2016.

[18] S. M. Stankov, R. Warnant, and K. Stegen, "Trans-ionospheric GPS signal delay gradients observed over mid-latitude Europe during the geomagnetic storms of October-November 2003," Adv. Space Res., vol. 43, no. 9, pp. 1314-1324, May 2009.

[19] D. P. Grubor, D. M. Šulić, and V. Žigman, "Classification of X-ray solar flares regarding their effects on the lower ionosphere electron density profile," Ann. Geophys., vol. 26, pp. 1731-1740, Jun. 2008

[20] J. A. Ferguson, Computer Programs for Assessment of Long-Wavelength Radio Communications, Version 2.0: User Guide. San Diego, CA, USA: Space and Naval Warfare Systems Center, 1998.

[21] J. R. Wait and K. P. Spies, "Characteristics Earth-ionosphere waveguide for VLF radio waves," Nat. Bureau Standards, Boulder, CO, USA, Tech. Note 300, 1964

[22] N. R. Thomson, "Experimental daytime VLF ionospheric parameters," J. Atmos. Terr. Phys., vol. 55, pp. 173-184, Feb. 1993.

[23] A. Kumar and S. Kumar, "Solar flare effects on D-region ionosphere using VLF measurements during low- and high-solar activity phases of solar cycle 24," Earth Planets Space, vol. 70, no. 1, Feb. 2018, Art. no. 29

[24] E. D. Schmitter, "Modeling solar flare induced lower ionosphere changes using VLF/LF transmitter amplitude and phase observations at a midlatitude site," Ann. Geophys., vol. 31, no. 4, pp. 765-773, Apr. 2013.

[25] A. Ishimaru, Electromagnetic Wave Propagation, Radiation, and Scattering. Hoboken, NJ, USA: Wiley, 2017.

[26] M. Radovanović, "Investigation of solar influence on the terrestria processes: Activities in Serbia," J. Geograph. Inst. Cvijic, vol. 68, no. 1, pp. 149-155, Apr. 2018.

[27] M. Todorović Drakul, V. M. Čadež, J. Bajčetić, D. B. L. C. Popović, and A. Nina, "Behaviour of electron content in the ionospheric D-region during solar X-ray flares," Serbian Astronomical J., vol. 193, pp. 11-18, Dec. 2016.

[28] G. Xu, GPS: Theory, Algorithms and Applications. Berlin, Germany: Springer, 2007. 